\title{
QUALITY IMPROVEMENT Improving the quality of electronic discharge summaries from medical wards: A quality improvement project
}

\author{
Authors: Glesni Davies, ${ }^{A}$ Stephanie Kean ${ }^{B}$ and Indrajit Chattopadhyay ${ }^{C}$
}

\section{Introduction}

The electronic discharge (e-discharge) summary forms an essential component of communication between secondary and primary care. However, its content and quality can often be substandard due to inadequate or inaccurate information.

\section{Method}

Two retrospective audits were completed with intervening e-discharge workshops. Local general practitioners were involved in identifying areas for improvement and assisted with the workshops. Crib sheets were emailed to all junior staff and posted on all medical wards.

Results

There was an improvement in the quality of e-discharges with particular improvements on the documentation of test results and patient progress and outcome. Those who attended the workshops produced better quality e-discharges and none recommended unnecessary actions for general practitioners.

Conclusions

E-discharge workshops are effective in improving the overall quality of discharge summaries from medical wards. Introduction of mandatory e-discharge training sessions during hospital induction and junior doctor rotations would be beneficial to teach this important yet challenging skill.

KEYWORDS: electronic discharge, e-discharge, discharge summary, quality, workshop

DOI: 10.7861/fhj.2020-0072

\section{Introduction}

The electronic discharge (e-discharge) summary forms an essential component of communication between secondary and primary care following a patient's discharge from the hospital. This may be the only source of information available to healthcare professionals and carers when the patient requires post-discharge

Authors: ${ }^{A}$ specialty trainee geriatrician, Glan Clwyd Hospital, Rhyl, UK; ${ }^{B}$ foundation trainee, Glan Clwyd Hospital, Rhyl, UK; ${ }^{C}$ consultant geriatrician, Glan Clwyd Hospital, Rhyl, UK care or review, or when they re-present to secondary care. Yet the content and quality of these discharge communications can be substandard and variable due to inadequate or inaccurate information, particularly for patients with complex clinical problems and long hospital stays requiring multiple interventions. Poor quality discharge summaries can lead to increased risk of patient care-related adverse events, undesirable outcomes and unplanned hospital re-admissions. ${ }^{1,2}$

E-discharges are usually completed by junior doctors (training and non-training grades) and other professionals like advanced nurse practitioners (ANPs), physician associates (PAs) and trainee ANPs who may have little training in this key skill and may rarely receive supervision or feedback. Other contributory factors may include lack of continuity of care, time and workforce constraints and poor understanding of the patients' diagnoses, interventions and clinical progress during their hospital stay.

There is clear evidence that e-discharges are favoured over traditional written discharges. ${ }^{3,4}$ However, there has been little research looking at how to ensure that the e-discharges that are generated are of adequate quality. Previous studies have demonstrated that teaching discharge summaries through a curriculum (including feedback and small group sessions) were effective in improving the quality of discharge communications. ${ }^{5,6}$ However, this appears to be more challenging in adult patients, particularly those with complex and multiple diagnoses and long lengths of stay.

While targeted and intensive educational interventions involving junior doctors may improve the quality of discharge communications, practical and sustainable strategies to maintain quality standards and engagement of junior doctors can still be challenging. ${ }^{7,8}$ As the Medicines Transcribing and e-Discharge (MTeD) system is being developed and integrated with the Welsh Clinical Portal digital workspace across NHS Wales, this project was undertaken in a busy district general hospital in north Wales to improve the quality of e-discharges from the medical wards.

\section{Method}

The plan, do, study, act (PDSA) cycle comprised two audits with interventions to address the gaps and drive improvement. Audit 1 was undertaken retrospectively and involved two assessors (trainee doctors) collecting data from the case notes of the 


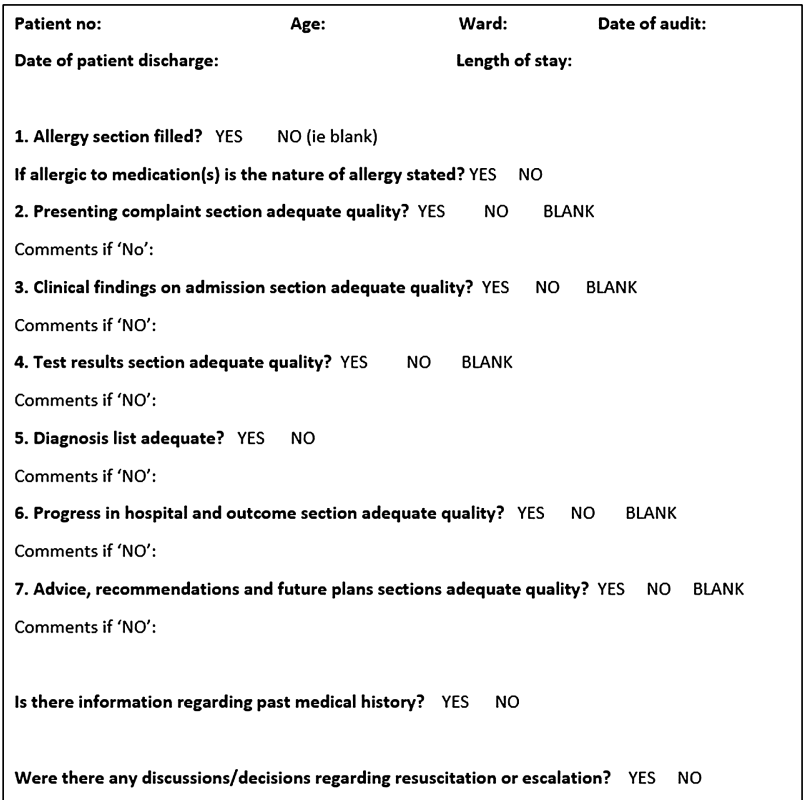

Fig 1. Audit pro forma for e-discharge.

previous six patients discharged from each of the 11 medical wards at Glan Clwyd Hospital during October and November 2019. The audit pro forma (Fig 1) consisted of seven questions about the quality of each e-discharge section with two additional questions on the inclusion of relevant past medical history, resuscitation decision and treatment escalation plans.

Following data collection, we collaborated with local GPs to ascertain their perspectives on anything that should or should not be included in an e-discharge summary. A particular focus was the 'advice and recommendations for GPs' section and a discussion surrounding examples of recommendations that were appropriate or inappropriate.

A series of e-discharge workshops were conducted during the trainees' weekly teaching sessions (foundation year-1, foundation year-2 and core medical/internal medicine trainees). Each workshop lasted an hour and involved feedback from audit 1 with best practice advice coupled with input from a local GP. This was followed by a practical session writing a discharge summary using our local e-discharge pro forma and a case example from the Royal College of Physicians learning resource materials. ${ }^{9}$ Feedback from the workshops was obtained and analysed. Following this, crib sheets were emailed to all junior medical staff and placed on all medical wards.

Audit 2 was conducted in February 2020 using the same pro forma as audit 1 , with one additional question on whether or not the e-discharge author had attended the workshop.

\section{Results}

Sixty-six case notes were audited for both audits 1 and 2 across 11 medical wards. A sub-group analysis of the e-discharges authored by those who had attended the workshop (33 e-discharges) was also undertaken.

Thirty-five doctors attended the workshop in total; $85 \%$ of these had not received previous training in writing e-discharges and $97 \%$ stated they would change their future practice based on these sessions. Feedback from the workshop was overall extremely positive.

The results (Table 1) showed improvements in the quality of most sections, particularly in the sub-group of e-discharges authored by those who had attended the workshops. 'Test results' and 'progress and outcome' sections showed significant improvements. Although there was an increase in the number of e-discharges with an adequate diagnosis, this improvement was not statistically significant.

In addition, there was no significant improvement seen in the quality of the 'advice and recommendations' section, however, there was a reduction in the number of job requests for GPs from $29 \%$ of e-discharges in audit 1 to $18 \%$ in audit 2 . For the e-discharges authored by the workshop attendees, only $9 \%(p=0.028$ when compared with audit 1$)$ had requested the GP to action something and these were all appropriate requests.

E-discharges authored by those who had attended the workshops demonstrated a significant improvement in the documentation of nature of allergy $(p=0.0027)$. This sub-group also demonstrated a significant improvement in achieving adequate quality in all sections $(p<0.001)$. However, there is

Table 1. Quality of e-discharges of all sections from audits and workshop

\begin{tabular}{|c|c|c|c|c|c|}
\hline & $\begin{array}{l}\text { Audit 1, } \\
n=66\end{array}$ & $\begin{array}{l}\text { Audit 2, } \\
n=66\end{array}$ & $\begin{array}{l}\text { p value (compared } \\
\text { with audit } 1 \text { ) }\end{array}$ & $\begin{array}{l}\text { Workshop } \\
\text { attendees, } n=33\end{array}$ & $\begin{array}{l}\mathrm{p} \text { value (compared } \\
\text { with audit } 1 \text { ) }\end{array}$ \\
\hline Allergies recorded & $86.0 \%$ & $86.0 \%$ & NS & $87.9 \%$ & NS \\
\hline Allergy nature stated & $4.76 \%$ & $35 \%$ & 0.016 & $50.0 \%$ & 0.0027 \\
\hline Presenting complaint adequate & $90.9 \%$ & $92.4 \%$ & NS & $97.0 \%$ & NS \\
\hline Clinical findings adequate & $90.9 \%$ & $86.3 \%$ & NS & $90.9 \%$ & NS \\
\hline Test results adequate & $54.5 \%$ & $84.8 \%$ & $<0.001$ & $90.9 \%$ & $<0.001$ \\
\hline Diagnosis adequate & $74.2 \%$ & $80.3 \%$ & NS & $84.8 \%$ & NS \\
\hline Progress and outcome adequate & $48.5 \%$ & $72.7 \%$ & $<0.01$ & $90.9 \%$ & $<0.001$ \\
\hline Advice and recommendations adequate & $86.4 \%$ & $83.3 \%$ & NS & $84.8 \%$ & NS \\
\hline All sections of adequate quality & $21.2 \%$ & $34.8 \%$ & NS & $54.5 \%$ & $<0.001$ \\
\hline
\end{tabular}


Table 2. Results of additional information

\begin{tabular}{llllll} 
& $\begin{array}{l}\text { Audit 1, } \\
\mathbf{n}=66\end{array}$ & $\begin{array}{l}\text { Audit 2, } \\
\mathbf{n}=66\end{array}$ & $\begin{array}{l}\text { p value (compared } \\
\text { with audit 1) }\end{array}$ & $\begin{array}{l}\text { Workshop } \\
\text { attendees, } \mathbf{n = 3 3}\end{array}$ & $\begin{array}{l}\text { p value (compared } \\
\text { with audit 1) }\end{array}$ \\
Past medical history documented & $31.8 \%$ & $53.0 \%$ & 0.016 & $67.0 \%$ & $<0.001$ \\
Resuscitation/escalation decision documented & $11.8 \%$ & $30.0 \%$ & NS & $40.0 \%$ & NS \\
\hline NS = not significant. & & & &
\end{tabular}

clearly still room for further improvement, with only $54.5 \%$ achieving adequate quality in all sections.

There was a significant improvement in the number of e-discharges containing information regarding past medical history (Table 2). However, we are still relatively poor at communicating resuscitation decisions and treatment escalation plans on e-discharges, which may be due to our hospital's practice of including a separate written document accompanying the e-discharge.

\section{Discussion}

Audit 1 showed notable inadequacies in documentation of relevant test results and progress of the patients in hospital including whether discussions were held in matters related to resuscitation, ceilings of care and treatment escalation plans (Table 1). Audit 1 also highlighted instances in $29 \%$ of e-discharges where requests were made to the GPs when the onus of executing some of those actions should have been on the patient's own team (Box 1). The reasons for shortfalls in e-discharge standards were likely to be manifold including lack of knowledge on expected standards, lack of skills and inability to grasp the patient's clinical journey from the documented notes due to complexity, lack of continuity of care, time pressure, low prioritisation and demand in the ward to maintain patient flow.

One of the major highlights of this quality improvement project was collaboration with the local GPs to ascertain their perspectives on the quality of e-discharges. They provided examples of what should and should not be included for GPs to action and what to be aware of, and they were also involved in the educational workshops with the junior doctors. The feedback from the workshops included several comments regarding how valuable the GP perspectives were.

Though there was an overall improvement in the quality of e-discharges in audit 2, particular improvements were noticed on the documentation of the quality of test results $(p<0.001)$ and patient progress and outcome $(p<0.01)$ with more e-discharges

\section{Box 1. Examples of inappropriate requests made to} general practitioners on e-discharges

Please chase anti-tissue transglutaminase antibodies

Review vitamin B12 bloods

Please re-check urea and electrolytes and magnesiumin 1 week

Please chase HIV results

Please arrange for Ferinject ${ }^{\circledR}$ once antibiotics finished

General practitioner kindly chase serum electrophoresis results

3/7 Phosphate Sandoz ${ }^{\circledR}$ prescribed; please check bloods on completion of this course containing information on past medical history. There was no significant improvement seen in the quality of the diagnosis section, primarily due to this section being left blank. Even the e-discharges authored by those who had attended the workshops had a blank diagnosis section in $15 \%$. Clearly this section is a key area and entering a diagnosis should be compulsory prior to electronic sign off.

Those who attended the workshops did produce better quality e-discharges and none recommended unnecessary or inappropriate actions for the GPs. However, none of the sections in audit 2 and for those who attended the workshops achieved $100 \%$, demonstrating the practical challenges as stated earlier and also the challenges surrounding variable human behaviour and learning curve.

The two audit cycles and the interventions were completed over a 4-month period, avoiding rotation of the majority of junior doctors between specialties. However, the project had some limitations. The e-discharge workshops mainly involved traininggrade junior doctors. As the audits were undertaken in the medical wards (including medical specialties), the e-discharge workshops did not include doctors from other non-medical specialties namely surgical, orthopaedics, ear nose and throat, obstetrics and gynaecology etc. In view of the practicalities, the workshops also did not involve other non-medical professionals involved in writing e-discharges, namely ANPs and PAs. However, the model for the e-discharge workshop is easily reproducible and can be replicated in other specialties and involving other healthcare professionals. In order for the quality of e-discharges to be consistently adequate, all staff completing e-discharges must be trained; therefore, introducing this as a mandatory session during hospital induction and junior doctor rotations seems reasonable. Another limitation is that patient perspectives were not obtained on matters related to e-discharge quality. This is something that should be considered as part of future projects.

\section{Conclusion}

E-discharge workshops with junior doctors are effective in improving the overall quality of discharge summaries from the medical wards. Improved documentation of test results, progress and outcome, and past medical history were particularly noticeable among those who attended the educational workshops, as well as a reduction of unnecessary requests for the GPs to action after discharge.

To maintain e-discharge standards, such workshops need to occur on a rolling basis in medical and non-medical specialties, also including other professionals who are involved in authoring e-discharges (ANPs, PAs and non-training grade doctors). Introduction of mandatory e-discharge training sessions during hospital induction and junior doctor rotations would also be beneficial to teach this important yet challenging skill. 


\section{References}

1 Van Walraven C, Seth R, Austin PC, Laupacis A. Effect of discharge summary availability during post-discharge visits on hospital readmission. J Gen Intern Med 2002;17:186-92.

2 Romagnoli KM, Handler SM, Ligons FM, Hochheiser H. Home care nurses' perceptions of unmet information needs and communication difficulties of geriatric patients in the immediate post-hospital discharge period. BMJ Qual Saf 2013;22:324-32.

3 Buckler LT, Teasdale C, Turner M et al. The patient-centered discharge-an electronic discharge process is associated with improvements in quality and patient satisfaction. J Healthc Qual 2017:39:136-43

4 Murphy SF, Lenihan L, Orefuwa F et al. Electronic discharge summary and prescription: improving communication between hospital and primary care. Ir J Med Sci 2017;186:455-9.

5 Key-Solle M, Paulk E, Bradford $K$ et al. Improving the quality of discharge communication with an educational intervention. Pediatrics 2010;126:734-9.

6 Myers JS, Jaipaul CK, Kogan JR et al. Are discharge summaries teachable? The effects of a discharge summary curriculum on the quality of discharge summaries in an internal medicine residency programme. Acad Med 2006;81:S5-8.

7 Cresswell A, Hart M, Suchanek $O$ et al. Mind the gap: Improving discharge communication between secondary and primary care. BMJ Qual Improv Rep 2015;u207936.w3197.

8 Shivji FS, Ramoutar DN, Bailey C, Hunter JB. Improving communication with primary care to ensure patient safety post-hospital discharge. Br J Hosp Med (Lond) 2015;76:46-9.

9 Royal College of Physicians. Improving discharge summaries learning resource materials. London: RCP, 2019. www.rcplondon. ac.uk/guidelines-policy/improving-discharge-summaries-learningresource-materials [Accessed 16 May 2020].

Address for correspondence: Dr Glesni Davies, Department of Geriatric Medicine, Glan Clwyd Hospital, Bodewlyddan, Rhyl LL18 5U], UK.

Email: gdav19@doctors.org.uk

Twitter: @GlesniDavies1 